\title{
Implementation of CBIR Method for Identification of Corn Disease Using Extreme Learning Machine
}

\author{
Hendri Purnomo \\ Department of Electrical \\ Engineering \\ University of Brawijaya \\ Malang, East Java, Indonesia
}

\author{
Panca Mudjirahardjo \\ Department of Electrical \\ Engineering \\ University of Brawijaya \\ Malang, East Java, Indonesia
}

\author{
Sholeh Hadi Pramono \\ Department of Electrical \\ Engineering \\ University of Brawijaya \\ Malang, East Java, Indonesia
}

\begin{abstract}
Various artificial neural network methods and digital image processing techniques have been applied in agriculture. Previous researchers have proposed methods for identification or detection of plant diseases, such as using K-NN, support vector machine (SVM), backpropagation and so on. The disease attack can reduce the amount of agricultural productivity and result in other material losses for farmers. Plant diseases can be observed from the physical and color changes of the affected parts such as roots, stems and leaves. However, with the development of computer vision technology, observations can be assisted by image processing methods and artificial neural networks, for automation in agriculture. This study proposes a method of applying digital image processing with artificial neural networks to identify corn plant diseases. The purpose of this research is as a comparative study of the proposed method with other conventional methods. The methods used are content based image retrieval (CBIR) and extreme learning machine (ELM). Content based image retrieval (CBIR) is the process of searching for an image in a database by comparing the features in the query image with the features that have been stored in the image database. Meanwhile, extreme learning machine (ELM) is an artificial neural network with one hidden layer or single hidden layer feedforward neural network (SLFNs). The feature extraction used consists of 3 features, namely color, shape, and texture feature extraction. The dataset consists of 3 types of images, namely images of healthy leaves, leaf spot disease, and rust disease. The ELM artificial neural network serves as a classifier that classifies the types of diseases based on the given feature extraction. The proposed system is able to identify the type of disease based on the pattern that appears on the leaf surface with the highest precision of 0.9922 .
\end{abstract}

Keywords: Canny edge detection, Color moment, CBIR, Extreme learning machine, GLCM

\section{INTRODUCTION}

The need for corn in Indonesia as a staple food other than rice is increasing every year. Besides being used as a staple food, corn can also be processed into various other products such as corn flour, corn oil, animal feed ingredients and so on. One of the ways to meet national corn needs is to increase the productivity of corn plants. But on the other hand, diseases that attack corn plants can reduce corn productivity and cause other material losses. The disease can be observed from changes in leaves, roots, and stems. Diseased plants if not treated immediately can inhibit plant growth and eventually die.

Observations of corn plant diseases can be observed directly through the five senses, namely the human eye. However, with the development of digital image processing technology and artificial neural networks can help in the development of agricultural automation in the identification of plant diseases. The advantage of using an artificial neural network is that it can overcome the problem of classifying corn plant disease images based on patterns that appear on the leaf surface through a number of calculation processes during learning and the use of large datasets can recognize patterns relatively easily by making complex relationships between layers in the network based on training data. [1][2]. The artificial neural network will conduct training on a given image pattern to form a model and certain rules as a reference, then based on the training data it can be used to recognize the pattern given in the test based on the reference to the training [3].
Previous research has carried out identification of corn plant diseases through the image of infected leaves, such as identifying corn leaves that are infected with rust and differentiating them from healthy corn leaves. The method used is to use a morphological operation with a threshold based on area. Based on the proposed method, diseased leaves were able to accurately quantify the degree of damage caused by rust spots [4].

Further research in identifying corn plant diseases is to use the bag of features method and the support vector machine (SVM) algorithm. The leaf images used were 2,000 images which were processed using the bag of features (bof) method and histogram statistics based on texture features. Then classified with multiclass support vector machine (SVM). Based on this research, the proposed method is able to provide an accuracy of $83.7 \%$ in identifying corn plant diseases [5].

The next research is to classify images based on similarity of features using the bag of features (bof) method which can generate features automatically. The steps taken consist of 4 stages, namely the stage of feature point location, feature extraction, visual word grouping, and classification using a support vector machine (SVM). The images used are divided into 3 types of images, namely RGB, greyscale, and segmented images. Each type is divided into 4 image classes, namely healthy leaf images, cercospora, rust disease and leaf blight where each class consists of 50 images. The results of this study indicate an accuracy of $82 \%$ for RGB images, $77 \%$ for grayscale images and $85 \%$ for segmented images [6]. 
Other researchers investigated the various uses of features in the classification of maize plant diseases. The features used are RGB color features, scale-invariant feature transform (SIFT), speeded up robust features (SURF), and Oriented FAST and rotated BRIEF (ORB). Performance evaluation of these features uses several machine learning algorithms such as support vector machines (SVM), Decision Tree (DT), Random forest (RF), and Naive Bayes (NB). With the number of image samples as many as 3,823 images which are divided into 4 classes of corn plant diseases. The evaluation results show that the use of RGB color features gives the best accuracy results in the classification of plant diseases [7].

The research proposed in this paper is to design a system using a combination of two methods, namely content based image retrieval (CBIR) and extreme learning machine (ELM) to identify corn plant diseases using the image of infected leaves. The proposed CBIR system uses three feature extraction methods, namely color feature extraction using color moment, texture feature extraction using gray level cooccurrence matrix (GLCM), and shape feature extraction using the canny edge detection method. While the ELM will function as a classifier that will determine the similarity of the two images between the query image and the image stored in the database.

\section{METHOD}

\subsection{Color Moment}

Color moment is a feature that can be used to distinguish images based on their color. The color distribution in an image can be interpreted as a probability distribution which is the basic assumption of the color moment. The calculation of the probability distribution used is the mean, standard deviation, and skewness [8].

A color space consists of three color components. For example, the RGB dimension space consists of three basic color components, namely Red (red), Green (green), and Blue (blue). Moment calculation is performed on each color component, so that it will produce nine moments as the identity of an image.

The HSV (hue, saturation, value) color space is a color space that represents the human senses of vision. The hue component represents the color, the saturation represents the level of color dominance, and the hue represents the brightness level, so this method can identify the color, the level of dominance and brightness. Hue values range from $0-$ 360 degrees, with color variations ranging from red to yellow, green, cyan, blue, to return to red [9].

To get the value of the color moment feature extraction, it can be seen in the diagram shown in Figure 1 [10].

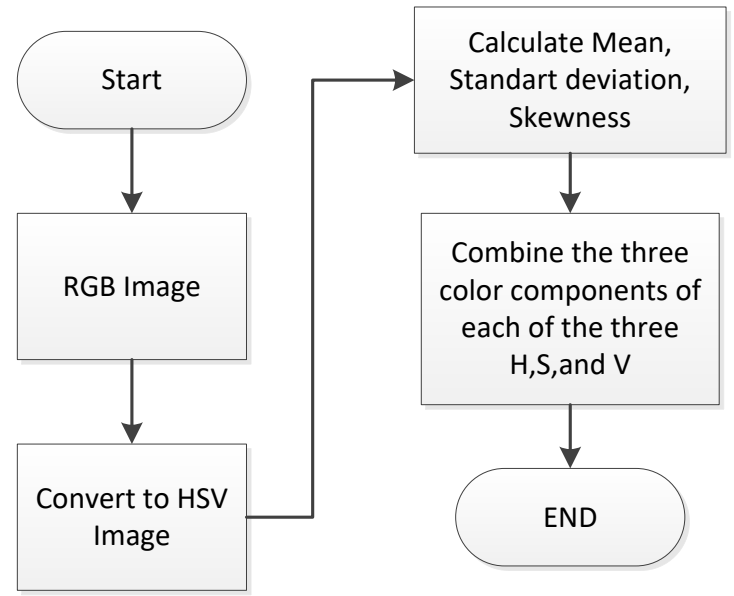

Figure 1. Color moment feature extraction flowchart

Calculation of color statistics on the color moment method to get the mean, standard deviation, and skewness is explained as follows [11]. The mean (mean) represents the distribution and is calculated using the equation:

$$
\mu=\frac{1}{M N} \sum_{i=1}^{M} \sum_{j=1}^{W} P_{i j}
$$

The standard deviation is obtained from the square root of the variance which expresses the distribution area,

$$
\sigma=\sqrt{\frac{1}{M N} \sum_{i=1}^{M} \sum_{j=1}^{M}\left(P_{i j}-\mu\right)^{2}}
$$

Skewness or inclination is a measure of asymmetry, calculated using the equation:

$$
\theta=\frac{\sum_{i=1}^{M} \sum_{j=1}^{N}\left(P_{i j}-\mu\right)^{3}}{M N \sigma^{3}}
$$

The data distribution will be skewed to the left if the skewness is negative, and vice versa. However, if the distribution is symmetrical, then the skewness coefficient will be zero.

\subsection{Grey Level Co-occurrence Matrix (GLCM)}

Haralick (1973) proposed a texture feature extraction method using a gray level co-occurrence matrix (GLCM) to explain partial patterns using 28 features. GLCM is calculated based on an order 2 matrix approach based on the relationship between pairs of two pixels in an image with a certain intensity, angle direction, and distance. The angles used in this method are $0^{\circ}, 45^{\circ}, 90^{\circ}$ and $135^{\circ}$. Of the 28 features presented, only 14 features that can be used are angular second moment (ASM), contrast, correlation, variance, inverse different moment (IDM), sum average, sum variance, sum entropy, entropy, difference entropy, information measures correlation. 1 and 2, and the maximal correlation coefficient [12]. However, Newsam and Kammath in their journal propose using only 5 features in texture feature analysis using GLCM, namely angular second moment (ASM)/energy, contrast, inverse different moment (IDM)/homogeneity, entropy, and correlation [13]. 
TABLE I

GLCM Feature Equation [14]

\begin{tabular}{|c|c|c|}
\hline No. & Fitur & Persamaan \\
\hline 1. & Energy & $P(\hat{i}, j)^{I}$ \\
\hline 2. & Contrast & $\sum_{\mathbb{L}=0} P_{L Q}(i-j)^{2}$ \\
\hline 3. & Homogeneity & $\sum_{\mathbb{L} \mathbb{W}=0}^{\mathbb{N}=4} \frac{P_{4}}{1+(i-j)^{2}}$ \\
\hline 4. & Entropy & $\sum_{\mathbb{Q}=0} P_{\mathbb{L}}\left(-\ln P_{\mathbb{U}}\right)$ \\
\hline 5. & Correlation & 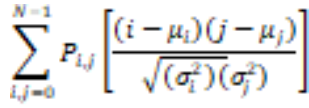 \\
\hline
\end{tabular}

Energy is the homogeneity value of an image. Contrast is the value of the variation of the gray level of pixels in an image. Entropy is a measure of the irregularity of the gray level in the image. And correlation is the value of the linear dependence between the gray level values in an image.

\subsection{Canny Edge Detection}

The canny edge detection method was first introduced in 1986 as an operator for edge detection based on optimization algorithms. The canny operator has three advantages and has become the standard for edge detection including :

- Better SNR (signal noise ratio) means that it is less possible to determine the edges and non-edges of an image, so that all edges can be detected properly.

- Better location performance, the detected edge is in the exact center of the edge or in the actual position.

- One edge corresponds to one response, one response to an actual edge [15].

The Canny algorithm consists of several steps, described in the form of a flowchart as follows [16]:

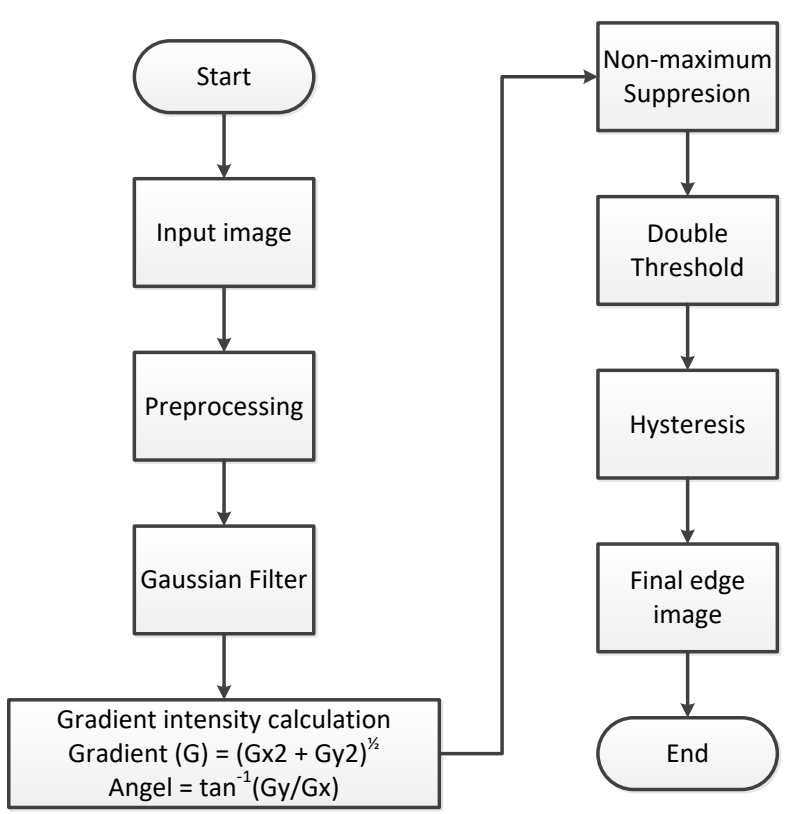

Figure 2. Canny edge detection feature extraction flowchart

1. Smoothing.

To reduce noise or smooth out an image, Canny uses a Gaussian filter with Eq.

$$
H_{i, j}=\frac{1}{2 \pi \sigma^{2}} e^{-\frac{\left(1-(k+1) j^{2}+\left(j-(k+1)^{2}\right.\right.}{2 \sigma^{2}}}
$$

Di mana :

$\mathrm{i}, \mathrm{j} \quad$ : the filter coordinates on the $\mathrm{x}$-axis and $\mathrm{y}$-axis, respectively.

k : Gaussian kernel size

$\sigma($ sigma $):$ standard deviation

2. Finding gradients magnitude and direction.

The magnitude of the gradients magnitude is determined by the equation:

$$
G=\sqrt{G_{x}^{2}+G_{y}^{2}}
$$

Sedangkan, nilai sudut (direction) di hitung dengan menggunakan persamaan :

$$
\theta=\tan ^{-1}\left(\frac{G_{x}}{G_{y}}\right)
$$

$G_{x}$ is the first derivative of the component on the horizontal axis, and $G_{y}$ is the first derivative of the component on the vertical axis. Value of $G_{x}$ and $G_{y}$ ditentukan menggunakan masking berikut. 


\begin{tabular}{|l|l|l|}
\hline 1 & 0 & -1 \\
\hline 1 & 0 & -1 \\
\hline 1 & 0 & -1 \\
\hline
\end{tabular}

(a)

\begin{tabular}{|l|l|l|}
\hline 1 & 1 & 1 \\
\hline 0 & 0 & 0 \\
\hline-1 & -1 & -1 \\
\hline
\end{tabular}

(b)
Figure 3. Derivative masking (a) $G_{x}$ and (b) $G_{y}$

\section{Non-maximum suppression}

This step is to localize the edges precisely. For that each pixel will be checked whether it is the edge of the maximum value in the surrounding local area. If the value of a pixel is the maximum value, it will be retained. On the other hand, if the pixel value is lower, it will be converted to a zero value. The value of the gradient is determined based on the direction of the angle which is divided into 4 groups [17]:

a. Horizontal with direction in the range $\left(-22.5^{\circ}, 22.5^{\circ}\right)$ or $(-$ $\left.157.5^{\circ}, 157.5^{\circ}\right)$

b. Vertical with direction in the range $\left(-112.5^{\circ},-67.5^{\circ}\right)$ or $\left(112.5^{\circ}, 67.5^{\circ}\right)$

c. +45 , with directions between $\left(-67.5^{\circ},-22.5^{\circ}\right)$ or $\left(112.5^{\circ}\right.$, $\left.157.5^{\circ}\right)$

d. -45 , with directions between $\left(-157.5^{\circ}, 112.5^{\circ}\right)$ or $\left(22.5^{\circ}\right.$, $\left.67.5^{\circ}\right)$

The illustration of the direction of the corner above is shown in Figure 4.

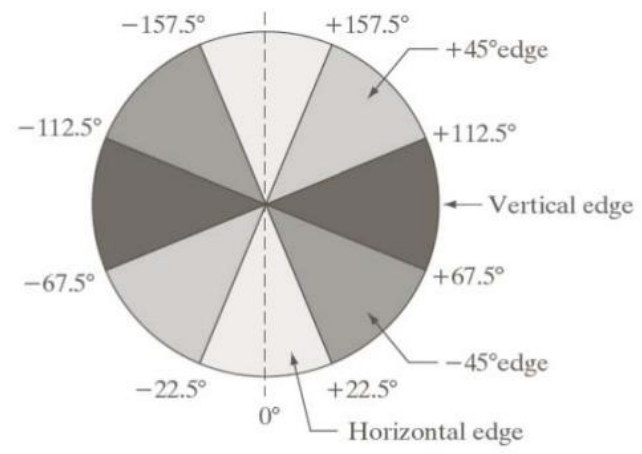

Figure 4. Range of angle direction of non-maximum suppression

4. Double thresholding

Potential edges are determined using thresholding, this is done to reduce the number of non-edge edges in the image. Thresholding consists of two T1 (upper limit) and T2 (lower limit). Edges with values $>$ T1 are strong edges. Edge values that are between $\mathrm{T} 2$ and $\mathrm{T} 1$ are weak edges. Meanwhile, values below the T1 threshold will be deleted.

5. Edge tracking by hysteresis thresholding.

Determination of the edge of the image is done based on its connectivity. If it is connected to a strong edge it is considered an edge, otherwise it is deleted [18].

\subsection{Content Based Image Retrieval (CBIR)}

Image retrieval (image retrieval) is the process of searching for an image with an input (query) in the form of an image. Specifically known as content based image retrieval (CBIR). This method was first proposed by Kato in 1992.
This method consists of two steps, namely feature extraction and feature matching. The features contained in the image will be extracted and stored in the database. The features used can be a single feature or more than one feature [19]. To get these features, the preprocessing stage is carried out by converting color and changing the image size. Furthermore, feature extraction is carried out in the form of color, shape, and texture features.

The content based image retrieval method can be described in the form of a flow chart as shown in Figure 5 [20].

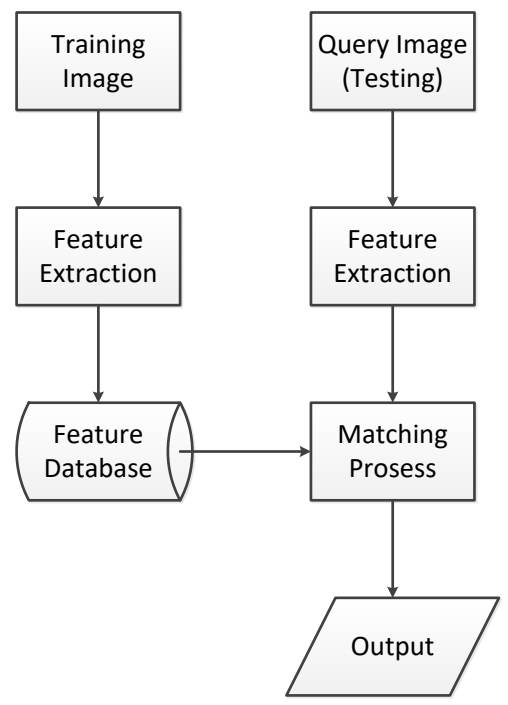

Figure 5. CBIR Flowchart

Determination of similarity between two images is done by calculating the feature distance. Distance is a common approach used for image search. Its function is to get the similarity between two features. The features obtained from the extraction of the query image are compared with those that have been stored in the database. The image with the closest distance is an image similar to the query image [21].

The use of the CBIR method is able to increase accuracy in image search when compared to text-based using keywords. The CBIR method is able to extract low-level image features automatically and measure image similarity by comparing features. Color, texture, and shape features are low-level features that are useful in representing and comparing images automatically [22].

\subsection{Extreme Learning Machine (ELM)}

Extreme learning machine (ELM) is a feedforward artificial neural network with a single hidden layer feedforward network (SLFNs). This algorithm was first proposed by Huang (2004) [23]. Generally, in other feedforward neural networks in order to produce high performance, the parameters used such as threshold values, weights, and activation functions must be appropriate to the system used. By using a gradient-based approach, all these parameters are changed iteratively resulting in low performance because they are trapped in the local minima.

In contrast to gradient-based ANN (artificial neural network), the use of input weights in the ELM algorithm is chosen randomly while the output weights are calculated analytically, so as to reduce the completion time and error values trapped 
in the local minima. This can improve the performance results of the ELM algorithm. The activation functions that can be used in ELM are linear and non-linear functions (sigmoid, sine, gaussian), and discrete or non-derivable functions [24].

The ELM method has been applied in the field of classification and researchers have obtained better and faster learning and generalization performance results compared to other gradient-based methods such as backpropagation. There are two parameters that need to be tested in using ELM as a classifier, namely the number of hidden neurons $(\mathrm{N})$ and the activation function $(\mathrm{F})$ [25].

SLFN with $\mathrm{L}$ hidden nodes can be represented by the following equation [26]:

$$
\begin{aligned}
& f_{L}(x)=\left(\sum_{i=1}^{L} G_{i}\left(x, a_{i}, b_{i}\right) \cdot \beta_{i}, \quad a_{i} \in R^{d}, b_{i}, \beta_{i} \in R\right. \\
& G_{i}\left(x, a_{i}, b_{i}\right)=g\left(a_{i}, x+b_{i}\right)
\end{aligned}
$$

$G_{i}(x)$ is the activation function at the i-th hidden node. $\Omega_{i j}$ is the input weight vector that connects the input layer to the $\mathrm{i}-\mathrm{th}$ hidden layer. $b_{i}$ is the bias weight of the $\mathrm{i}$-th hidden layer. $\beta_{i}$ is the output weight.

Equation (7) above can be simplified into the following equation:

$$
\beta=H^{+} T
$$

$T=\left[t_{1, \ldots, x} t_{N}\right]^{T} . H^{\Psi}$ is the inverse Moore-Penrose matrix H. Thus, the calculation of the output weight can be carried out by means of a mathematical transformation, to avoid lengthy training where the parameters are adjusted iteratively with several appropriate training parameters [27].

The ELM algorithm can be briefly explained as follows :

1) Randomly assign hidden node parameters. For example for input weight $a_{i}$ and bias $b_{i} . \mathrm{i}=1, \ldots, \mathrm{L}$

2) Calculate the hidden layer output matrix $\mathbf{H}$.

3) Calculate the output weight vector with equation (9).

\section{PROPOSED SYSTEM}

The CBIR system consists of two stages, namely the training and testing stages. The training phase consists of the configuration of the neural network responsible for studying the features of the extracted image, the learning process is carried out using the ELM algorithm. Furthermore, the network stores the rules that have been learned in the database. The rules are made for comparison and decision making. The comparison is done by comparing the query image with the image that has been stored in the database at the training stage. The neural network makes decisions according to the similarity between the two features of the query image and the database.

The testing stage is the stage in the image query. The image to be entered is first carried out in the preprocessing and feature extraction stages. At this stage the ELM network acts as a selective classifier in determining the image that is most similar to the image stored in the database. So that it can identify accurate and similar images efficiently according to the image query given [28]

The initial stage before the training and testing process is carried out in the initial stages of basic image processing for all images, both on training images and test images. The preprocessing stage is done with color conversion, and image resizing. Then the next step is feature extraction in the form of color, texture, and shape features. Color extraction is done by using a color moment. Color feature extraction is obtained by converting the image in RGB space to HSV and then performing statistical calculations such as mean, standard deviation, and skewness. Texture feature extraction was obtained using the gray level co-occurrence matrix (GLCM) method. The features used consist of angular second moment (ASM), contrast, inverse different moment (IDM), entropy, and correlation. The shape features are obtained by edge detection using the canny edge detection method.

The following is a block diagram of the proposed research procedure.

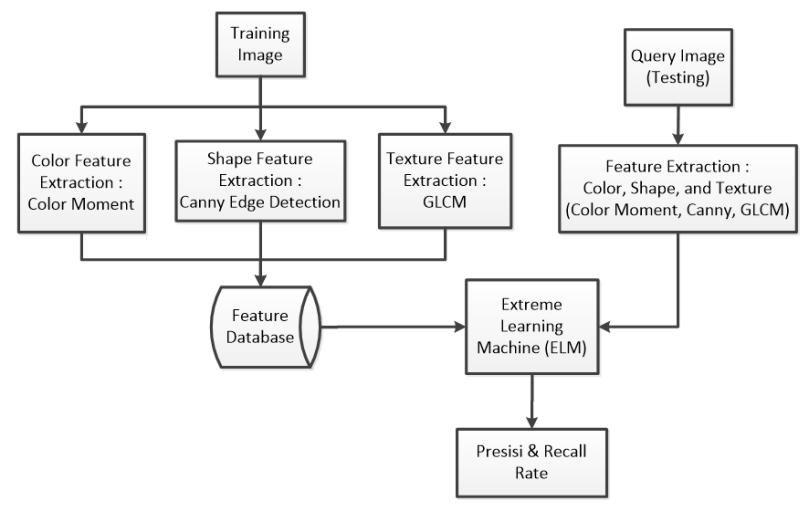

Figure 6. Flowchart of the proposed research.

\section{RESULT AND DISCUSSION}

The dataset used is secondary data obtained from https://github.com/charul97/Plant-disease-detection [29]. The dataset consists of 3 image categories, namely healthy leaves, common rust and leaf spot disease (cercospora). Figure 7 is an example of the image used.

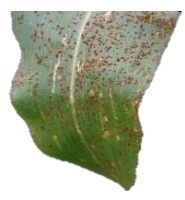

a. Common rust

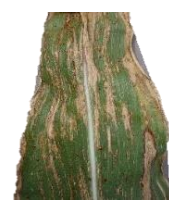

b. Cercospora

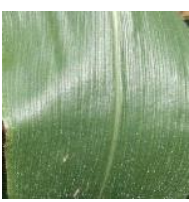

c. Healthy
Figure 7. Example of image dataset

The amount of data is divided into 2 parts, namely training data as many as 800 images and test data as many as 400 images. 
International Journal of Computer Applications Technology and Research

Volume 11-Issue 02, 14-21, 2022, ISSN:-2319-8656

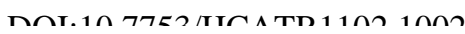

Table III

The value of precision and recall of system test results

\begin{tabular}{cccccccccc} 
& & \multicolumn{7}{c}{ Number of Hidden Nodes } \\
\cline { 3 - 10 } Activation function & 25 & 50 & 75 & 100 & 125 & 150 & 175 & 200 \\
\hline \multirow{2}{*}{ Tanh } & Precision & 0.9663 & 0.9786 & 0.9754 & 0.9732 & 0.9795 & 0.9834 & 0.9836 & 0.9699 \\
& Recall & 0.9611 & 0.9678 & 0.9744 & 0.9778 & 0.9833 & 0.9833 & 0.9778 & 0.9689 \\
\multirow{2}{*}{ Sine } & Precision & 0.7634 & 0.8851 & 0.9196 & 0.9363 & 0.9323 & 0.9326 & 0.9475 & 0.942 \\
& Recall & 0.7489 & 0.8833 & 0.9244 & 0.9344 & 0.9311 & 0.9333 & 0.9456 & 0.9467 \\
\multirow{2}{*}{ Tribas } & Precision & 0.8253 & 0.9159 & 0.9086 & 0.9275 & 0.9544 & 0.9657 & 0.9576 & 0.9663 \\
& Recall & 0.8267 & 0.9133 & 0.91 & 0.9244 & 0.9467 & 0.9644 & 0.9544 & 0.9589 \\
\multirow{2}{*}{ Hardlim } & Precision & 0.8195 & 0.9052 & 0.9274 & 0.9358 & 0.9559 & 0.9442 & 0.9441 & 0.9268 \\
& Recall & 0.8189 & 0.9 & 0.9289 & 0.9322 & 0.9567 & 0.9422 & 0.9456 & 0.9244 \\
\multirow{2}{*}{ Rbf } & Precision & 0.9922 & 0.9912 & 0.9845 & 0.966 & 0.9606 & 0.9369 & 0.9298 & 0.9429 \\
& Recall & 0.9911 & 0.9922 & 0.9822 & 0.96 & 0.9556 & 0.9344 & 0.9233 & 0.9411 \\
\hline
\end{tabular}

The input image (query) given in the form of an RGB image is carried out in a preprocessing stage in the form of conversion to gray scale, resizing and rescale then the feature extraction process using color moment, GLCM, and canny edge detection. The ELM classifier will compare the features that have been extracted in the database with the input image (query) and determine the type of disease based on its features.

The system for identifying corn plant diseases was made using the python programming language and using the google collaborative application. System testing is done by changing the value of the hidden node by using five types of activation functions as a comparison of the level of precision. The number of hidden nodes used ranges from 25 - 200 hidden nodes, while the types of activation functions consist of 5 types, namely hyperbolic tangent (tanh), sine, triangular basis (tribas), hard limit (hardlim), and radial basis function (Rbf). Meanwhile, for feature extraction using GLCM, the angle used is $45 \mathrm{o}$ with a neighboring distance of 1 pixel.

Effective performance measurement is needed as an evaluation of the algorithm used in designing the image retrieval application. The performance measurement of the CBIR system can be known through the level of precision and recall rate based on the truth table.

Precision is a comparison of the number of images that are really relevant. A good precision is worth 1.0. Precision can be calculated using equation (10):

Precision $=\frac{\text { True Positive }(T P)}{\text { True Positive }(T P)+\text { False Positive }(F P)}$

Recall rate is the ability of the system to select all images that have true values and not select images that have false negative values. The recall rate can be calculated using equation (11):

Recall $=\frac{\text { True positive }(T P)}{\text { True Positive }(T P)+\text { False Negative }(F N)}$

The first experiment was carried out using 25 hidden nodes with an activation function of the radial basis function (rbf), the results of the system test using the truth table as shown in Table II.
TABLE II

Truth Table System Test Results

\begin{tabular}{cccc}
\hline \multirow{2}{*}{ Actual } & \multicolumn{3}{c}{ Prediction } \\
\cline { 2 - 4 } & Cercos & Healthy & Rust \\
\hline Cercospora & 98 & 2 & 0 \\
Healthy & 1 & 149 & 0 \\
Common rust & 0 & 0 & 150 \\
\hline
\end{tabular}

In Table II above, the system has succeeded in identifying 150 common rust images, 98 images of leaf spot disease (cercospora) and 2 images identified as healthy leaf images. As for healthy leaf images, the system was able to identify 149 images and 1 image was identified as leaf spot disease (cercos).

The values in table II above are the number of images that have been identified by the system according to their respective classes, namely images of healthy leaves (healthy), leaf spot disease (cercospora), and rust disease (common rust), then by testing using five types of functions. Different activation and number of hidden nodes in each test with a value range of 25 - 200 hidden nodes obtained precision and recall values as shown in Table III.

Based on Table III above, it is known that the precision value of the system is in the range of values between 0.7634 0.9922 . While the recall value is in the range of $0.7489-$ 0.9922 . The best precision value among all tests is 0.9922 and the recall is 0.9911 using the radial basis function (Rbf) activation function with a total of 25 hidden nodes. In Table III it also appears that the precision value of the test with the tanh function shows a value that tends to be stable when compared to the rbf function which has decreased in the use of the 200 hidden nodes. This could be due to changes in the structure of the parameters used by machine learning which resulted in overfitting or underfitting resulting in fluctuating changes in value.

If with the addition of the structure, the value becomes high, then the system is underfitting. On the other hand, if the addition of the value structure becomes low, then the system is overfitting. Therefore, the extreme learning machine algorithm needs to adjust the parameters used in each test, so that the best value is obtained [30]. Based on Table III, the average value of the activation function can be seen in Figure 8 . 


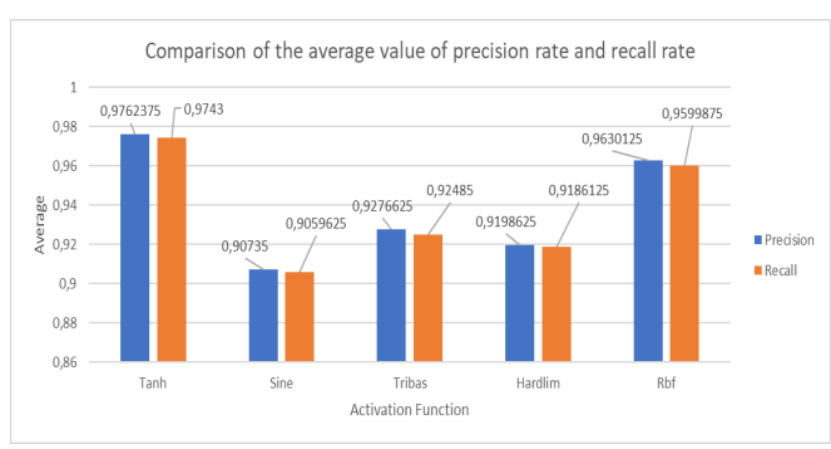

Figure 8. Comparison of the average precision and recall rate

Figure 8 shows the comparison of the average number of system precision and recall rate. In the graph, it can be seen that the Tanh function gives a higher average result than the other activation functions. However, testing using the radial basis function (rbf) with 25 hidden nodes gave the highest results compared to testing using the tanh activation function.

\section{CONCLUSION}

A plant disease identification system using content based image retrieval (CBIR) and extreme learning machine (ELM) algorithms has been carried out. The dataset used is an image of leaves affected by rust and leaf spot disease, as well as leaves that are not affected by the disease. Based on the results of testing the proposed system, the activation function of the radial basis function (rb) and the number of hidden nodes 25 gave the highest results compared to the other four types of activation functions, namely hyperbolic tangent (tanh), sine, triangular basis (tribas), and hard limit (hardlim). ). The system is able to identify the type of corn plant disease with a precision level of 0.9922 . This is supported by the use of three types of feature extraction, namely color, shape, and texture features in recognizing types of corn plant diseases using the image retrieval method and classified by the extreme learning machine algorithm. Further research needs to be tested with the addition of other types of plant diseases and different datasets. Also, optimization with certain methods so that a stable precision value is obtained in each test.

\section{REFERENCES}

[1] Kusumadewi, S. (2004). Membangun Jaringan Syaraf Tiruan: Menggunakan Matlab \& Excel Link. (F. W. Nurwiyati (ed.); Pertama). Graha Ilmu.

[2] Sholeh Hadi Pramono, Rohmatillah, M., Maulana, E., Hasanah, R. N., \& Hario, F. (2019). Deep LearningBased Short-Term Load Forecasting for Supporting Demand Response Program in Hybrid Energy System. Energies, 12(17), 1-16. https://doi.org/10.3390/en12173359

[3] Pangaribuan, Y., \& Sagala, M. (2017). Menerapkan Jaringan Saraf Tiruan untuk Mengenali Pola Huruf Menggunakan Metode Perceptron. Teknik Informatika Unika St. Thomas (JTIUST), 02(02), 53-59.

[4] Yadav, A., \& Dutta, M. K. (2018). An automated image processing method for segmentation and quantification of rust disease in maize leaves. 2018 4th International Conference on Computational Intelligence \&
Communication $\quad$ Technology
https://doi.org/10.1109/ciact.2018.848012

[5] Aravind, K. R., Raja, P., Mukesh, K. V, Aniirudh, R., Ashiwin, R., \& Szczepanski, C. (2018). Disease Classification in Maize Crop using Bag of Features and Multiclass Support Vector Machine. 2018 2nd International Conference on Inventive Systems and Control (ICISC), Icisc, 1191-1196.

[6] Syarief, M., Prastiti, N., \& Setiawan, W. (2019). Maize leaf disease image classification using bag of features. JURNAL INFOTEL, $11(2), \quad 48$ https://doi.org/10.20895/infotel.v11i2.428

[7] Kusumo, B. S., Heryana, A., Mahendra, O., \& Pardede, H. F. (2018). Machine learning-based for automatic detection of corn-plant diseases using image processing. 2018 International Conference on Computer, Control, Informatics and Its Applications (IC3INA). https://doi.org/10.1109/ic3ina.2018.8629507

[8] Maheswary, P., \& Srivastav, N. (2008). Retrieving similar image using color moment feature detector and K-means clustering of remote sensing images. Proceedings of the 2008 International Conference on Computer and Electrical Engineering, ICCEE 2008, 821824. https://doi.org/10.1109/ICCEE.2008.114

[9] Indriani, O. R., Kusuma, E. J., Sari, C. A., Rachmawanto, E. H., \& Setiadi, D. R. I. M. (2017). Tomatoes classification using K-NN based on GLCM and HSV color space. Proceedings - 2017 International Conference on Innovative and Creative Information Technology: Computational Intelligence and IoT, ICITech 2017, 1-6. https://doi.org/10.1109/INNOCIT.2017.8319133

[10] Das, S., Rudrapal, D., \& Sarkar, R. (2012). An Efficient Method for Content Based Image Retrieval Using Color Moment and Texture Descriptors. Nternational Conference on Computing, Communication and Information Technology, Icccit, 123-127.

[11] Kadir, A., \& Susanto, A. (2013). Teori dan Aplikasi Pengolahan Citra Digital. JogyakartaAndi2013.

[12] Haralick, R. M., Shanmugam, K., \& Dinstein, A. I. (1973). Textural Features for Image Classification. IEEE Transactions On Systems, Man, And Cybernetics., SMC3(6), 610-621. https://doi.org/1

[13] Newsam, S. D., \& Kamath, C. (2005). Comparing Shape and Texture Features for Pattern Recognition in Simulation Data. Image Processing: Algorithms and Systems IV, 5672(SPIE-IS\&T), 106-117.

[14] P.S, S. K., \& V.S, D. (2016). Extraction of texture features using GLCM and shape features using connected regions. International Journal of Engineering and Technology, 8(6), 2926-2930. https://doi.org/10.21817/ijet/2016/v8i6/160806254

[15] Lumchanow, W., \& Udomsiri, S. (2019). Combination of GLCM and KNN classification for chicken embryo development recognition. ECTI DAMT-NCON 2019 4th International Conference on Digital Arts, Media and Technology and 2nd ECTI Northern Section Conference on Electrical, Electronics, Computer and 

Telecommunications $\quad$ Engineering,
https://doi.org/10.1109/ECTI-NCON.2019.8692272

[16] Setiawan, B. D., Rusydi, A. N., \& Pradityo, K. (2017). Lake Edge detection using canny algorithm and Otsu thresholding. 2017 International Symposium on Geoinformatics (ISyG). https://doi.org/10.1109/isyg.2017.8280676

[17] M. K. Monir Rabby, B. Chowdhury and J. H. Kim, "A Modified Canny Edge Detection Algorithm for Fruit Detection \& Classification," 2018 10th International Conference on Electrical and Computer Engineering (ICECE), 2018, pp. 237-240, doi: 10.1109/ICECE.2018.8636811

[18] Ramamurthy, B., \& Chandran, K. R. (2011). Content based Image Retrieval for Medical Images using Canny Edge Detection Algorithm. International Journal of Computer Applications, 17(6), 32-37. https://doi.org/10.5120/2222-2831

[19] Patil, J. K., \& Kumar, R. (2016). Analysis of content based image retrieval for plant leaf diseases using color, shape and texture features. Engineering in Agriculture, Environment and Food, 10(2), 1-10. https://doi.org/10.1016/j.eaef.2016.11.004

[20] Butt, S. M., \& Tariq, M. U. (2013). Visual Feature Extraction for Content-Based Image Retrieval. International Journal of Academic \& Scientific Research, 1(3), 1-10. https://doi.org/10.1007/978-1-4899-79933_162-2

[21] Deng, C. X., Bai, T. T., \& Geng, Y. (2009). Image edge detection based on wavelet transform and canny operator. 2009 International Conference on Wavelet Analysis and Pattern Recognition, ICWAPR 2009, 2(July), $355-359$. https://doi.org/10.1109/ICWAPR.2009.5207469

[22] Mary, I. T. B., Vasuki, A., \& Manimekalai, M. A. P. (2017). An Optimized Feature Selection CBIR Technique using ANN. International Conference on Electrical, Electronics, Communication Computer Technologies and Optimization Techniques, ICEECCOT 2017,

$470-477$. https://doi.org/10.1109/ICEECCOT.2017.8284550

[23] Huang, G. Bin, Zhu, Q. Y., \& Siew, C. K. (2004). Extreme Learning Machine: A New Learning Scheme Of Feedforward Neural Networks. IEEE International Conference on Neural Networks - Conference Proceedings, 2, 985-990. https://doi.org/10.1109/IJCNN.2004.1380068

[24] Sönmez, Y., Tuncer, T., Gökal, H., \& Avci, E. (2018). Phishing Web Sites Features Classification Based on Extreme Learning Machine. 6th International Symposium on Digital Forensic and Security, ISDFS 2018 - $\quad$ Proceeding, https://doi.org/10.1109/ISDFS.2018.8355342

[25] Li, F. C., Wang, P. K., \& Wang, G. E. (2009). Comparison of the Primitive Classifiers with Extreme Learning Machine in Credit Scoring. IEEM 2009 - IEEE International Conference on Industrial Engineering and Engineering Management, 685-688. https://doi.org/10.1109/IEEM.2009.5373241
[26] Jiexiong Tang, Student Member, IEEE, Chenwei Deng, Senior Member, I., \& and Guang-Bin Huang, Senior Member, I. (2016). Extreme Learning Machine for Multilayer Perceptron. IEEE Transactions On Neural Networks And Learning Systems, 27(4), 809-821. https://doi.org/10.1109/TNNLS.2015.2424995

[27] Ding, S., Xu, X., \& Nie, R. (2013). Extreme Learning Machine and Its Applications. Neural Computing and Applications, 25(3-4), 1-8 https://doi.org/10.1007/s00521-013-1522-8

[28] Nagathan, A., Jitendranath, M., \& Mungara. 2014. Content-Based Image Retrieval System using FeedForward Backpropagation Neural Network.

[29] Smart India Hackathon. 2018. Smart India Hackathon 2k18 Project For Detecting Plant Disease Based On Images Of Plant Leaves Having Disease. https://github.com/charul97/Plant-disease-detection (diakses tanggal 1 February 2020)

[30] Anam, K., Avian, C., \& Nuh, M. (2020). Multilayer Extreme Learning Machine for hand movement prediction based on electroencephalography. Bulletin of Electrical Engineering and Informatics, 9(6), 2404-2410. https://doi.org/10.11591/eei.v9i6.2626 